\title{
Design and Manufacturing of Electromechanical Chip for Rapid Measurement of IgG1 Antibody in Cell-Culture Supernatant
}

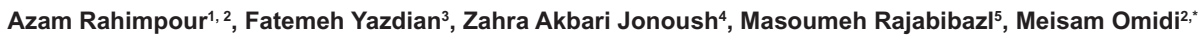 \\ ${ }^{1}$ Medical Nano-Technology \& Tissue Engineering Research Center, School of Advanced Technologies in Medicine, Shahid Beheshti University of Medical Sciences, \\ Tehran, Iran \\ ${ }^{2}$ Department of Tissue Engineering and Regenerative Medicine, School of Advanced Technologies in Medicine, Shahid Beheshti University of Medical Sciences, \\ Tehran, Iran
}

${ }^{3}$ Faculty of New Science and Technology University of Tehran, Tehran, Iran

${ }^{4}$ Department of Immunology, Shahid Sadoughi University of Medical Sciences \& Health Services, Yazd, Alem Sq., Safaieh, Iran

${ }^{5}$ Department of Medical Biochemistry, School of Medicine, Shahid Beheshti University of Medical Sciences, Tehran, Iran

*Corresponding author: Omidi M, Medical Nano-Technology \& Tissue Engineering Research Center, School of Advanced Technologies in Medicine, Shahid Beheshti University of Medical Sciences, Tehran, Iran; E-mail: m_omidi@sbmu.ac.ir

Received: Jul 27, 2016; Accepted: Aug 26, 2016; Published: Sep 22, 2016

Copyright: (C) 2016 Omidi et al. This is an open-access article distributed under the terms of the Creative Commons Attribution License, which permits unrestricted use, distribution, and reproduction in any medium, provided the original author and source are credited.

\begin{abstract}
In this study, we have used arrays of piezoresistive nanomechanical membrane resonators (NMRs) to detect IgG1 antibody. The NMR consists of a membrane suspended by four piezoresistive sensing components. Unlike conventional cantilever mass sensors, our sensors retain a uniform mass sensitivity over the membrane surface. According to the experiments, it was revealed that NMRs have mass sensitivities in the order of $25 \mathrm{~Hz} / \mathrm{pg}$ in air and $1.8 \mathrm{~Hz} / \mathrm{pg}$ in liquid. This matter allows them to detect IgG1 over a wide range of concentrations from $100 \mathrm{pg} / \mathrm{mL}$ to $10 \mu \mathrm{g} / \mathrm{mL}$ in a background of human serum albumin (HSA) at $1 \mathrm{mg} / \mathrm{ml}$. This indicates the fact that the self-sensing NMR approach is beneficial for detecting disease markers. Moreover, the performance of the NMR was compared with other detection methods, and the results indicated a better performance for NMR.
\end{abstract}

Keywords: Piezoresistive; Nanomechanical membrane resonators; Stem cell; IgG1 antibody

\section{Introduction}

Although the development of clinical sensors and biosensors has increased in recent years, improvements in sensitivity, selectivity, limits of detection, fast response, and miniaturization are yet to be attained. Health care appears to provide the best opportunity for sensor development. Among the wide range of different sensors and biosensors, microelectromechanical system (MEMS) biosensor is one of the best options for use in the clinical field, due to their high sensitivity and selectivity, portability, and rapid response time [1-3].

The central element in many traditional mechanical biosensors is a small cantilever that is sensitive to the biomolecule of interest. It is possible to operate microcantilever sensors in two different modes, i.e., cantilever bending (surface-stress method) and resonance-response variation (microbalance method). In the static mode, the induced surface stress that is due to the presence of the adsorbates results in a deflection in the cantilever [4], while in the dynamic mode, the adsorbates change the resonance frequency of a cantilever due to mass loading [5]. A sensitive readout system is crucial for monitoring the deflection of cantilevers. For this reason several readout methods have been presented. The most extended readout methods for biosensing are optical and piezoresistive ones. The optical method is simple to implement and shows a linear response with subangstrom resolution and also is currently the most sensitive method. This method is employed for detecting the cantilever deflection in most studies [5,6-8]. Nevertheless, the optical-detection mechanism presents some disadvantages, for example, bulky, time-consuming laser alignment on each cantilever and low applicability for large one- or two-dimensional arrays, and the difficulty of performing measurements in opaque liquids, such as blood, may hinder the potential application of this method for actual applications.

Among electrical techniques, piezoresistive detection is the most widespread. This detection requires the integration of a piezoresistive element during the fabrication of the nanomechanical system, which is usually located near the clamping regions. The change of resistance due to the motion-induced strain in the piezoelectric region is measured by a Wheatstone bridge. The piezoresistive sensing method is known as a good alternative for optical detection in biosensing applications. The benefit of this method is that the principle works well in both liquid and gas phases, and large arrays can be realized and read out. Also, the technique is applicable for static as well as dynamic measurements [9-12]. Although piezoresistive cantilevers have proven to be highly beneficial detection methods, without effective mechanical amplification schemes, their sensitivity is far below that of optical methods. In order to overcome this problem, several researches have focused on applying structural modification, such as making a through hole [13], patterning the cantilever surface [14], or variation of geometrical parameters (e.g., length, width, and overall shapes) $[15,16]$. Although all these methods have proven to improve the sensitivity of piezoresistive cantilevers for displacement detection, they have still not yielded significant stress amplification to make piezoresistive detection comparable to the optical approach, which can be due to the fact that all these approaches rely on suppressing one of the isotropic stress components. Analytical consideration of strain-amplification schemes for sensing applications based on the strategies of the constriction and double lever geometries [17] has resulted in the introduction of a nanomechanical membrane, which has shown a considerable improvement in amplifying piezoresistive 
Citation: Rahimpour A, Yazdian F, Jonoush ZA, Rajabibazl M, Omidi M (2016) Design and Manufacturing of Electromechanical Chip for Rapid Measurement of IgG1 Antibody in Cell-Culture Supernatant. Biol Med (Aligarh) 8: 340. doi:10.4172/0974-8369.1000340.

detection signals. Yoshikawa et al. [18] have experimentally evaluated a prototype nanomechanical membrane, and the results have illustrated a significant sensitivity for piezoresistive cantilevers. In comparison with the standard piezoresistive cantilever, this study demonstrated a factor of more than 20 times higher sensitivity than that obtained with a standard piezoresistive cantilever.

Additionally, it has demonstrated that the change in resonant frequency is dependent not only on the mass of the attached particle but also on the position on the beam, so cantilevers are not always the most sensitive geometries [3]. Waggoner et al. [19] have used arrays of nanomechanical resonators to detect prostate-specific antigens (PSAs). They have shown that membrane resonators have a relatively uniform frequency response for the mass of bound material located anywhere across its central paddle because of its unique resonant mode shape. In another work, due to uniform mass sensitivity, Park et al. [20] have used membrane resonators for characterizing the biophysical properties and growth of adherent human colorectal carcinoma cells (HT29).

In this work, we used a unique array of piezoresistive nanomechanical membrane resonators' (NMRs) mass sensors for identifying the IgG1 antibody. A direct nanomechanical response of a microfabricated selfsensing NMR was used for the dynamic detection of antigen-antibody specific binding. After injecting the IgG1-antibody target, as a model biocontent, the resonant-frequency shift was carefully analysed, and the feasibility of the piezoresistive membrane resonators for biosensing was discussed in terms of device-performance measures such as sensitivity, accuracy, and specificity. At the end, the results were compared with a standard cantilever.

\section{Theoretical Background}

The mass of a target sample attached to a resonant-mass sensor can be determined by measuring changes in the resonant frequency of the sensor. The resonant frequency of the sensors, $f_{0}$, is inversely proportional to the square root of its total mass $\left(f \propto \sqrt{D / m_{0}}\right)$. Hence, by measuring the resonant frequency, the mass of the sensor and the target sample can be measured through time. Cantilever structures have been widely used as resonant-mass sensors. However, conventional cantilever sensors (Figure 1a) exhibit severe nonuniform mass sensitivity, i.e., the location of the added mass relative to the free end determines the mass sensitivity or the ratio of the resonantfrequency shift to the actual mass [21]. Thus, mass detection can be falsely reported as a change in mass because of the nonuniform mass sensitivity of a cantilever. In this work, a unique sensor structure for uniform mass sensitivity was developed for accurate measurement of the biomass. Because the mass sensitivity is linearly proportional to the square of the vibration amplitude of the sensor structure, a four beam-spring sensor structure (Figure 1a) was designed to minimize the variation of the vibration amplitude across the vibrating platform.

The harmonic excitation of nanomechanical resonators can induce stress [22]. In the piezoresistive micromechanical/nanomechanical sensors, the electrical resistivity of a piezoresistive film varies with the applied stress. The resistance of the silicon $(\mathrm{Si})$ piezoresistor is a function of stress and the orientation of the piezoresistors. The relation between resistivity and stress can be expressed as [23]:

$$
\left[\frac{\Delta R}{R_{0}}\right]=\{\pi\}[\sigma]
$$

(a)

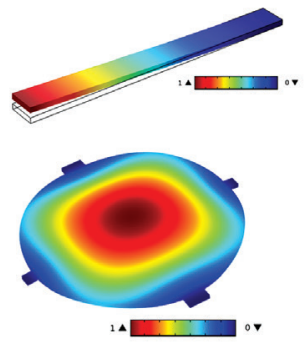

(b)
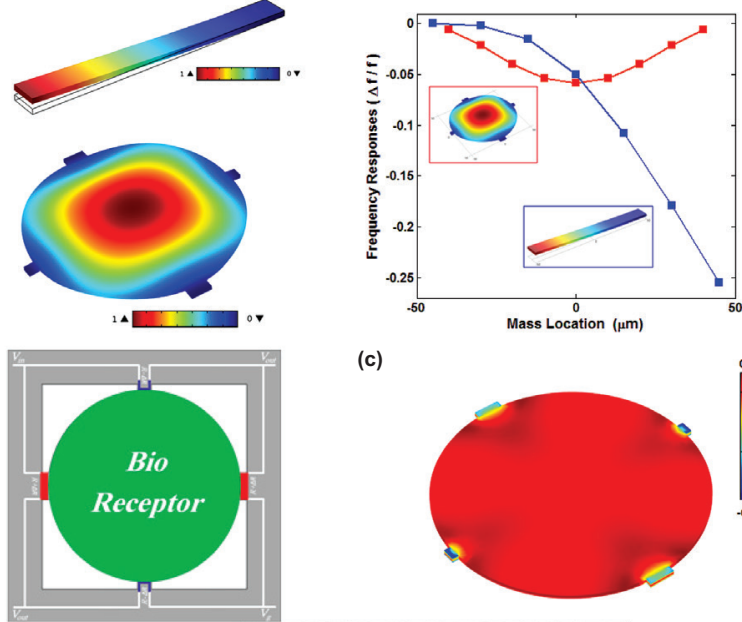

(c)

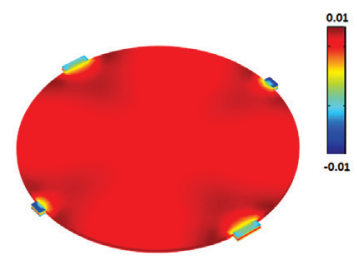

(d)

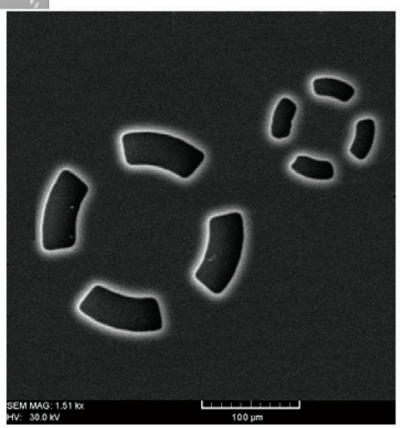

Figure 1: Finite element analyses (FEAs) using COMSOL Multiphysics 4.2. (a) The first mode of resonance is shown with the mass sensitivity. Modal analysis of cantilevers in a liquid via finite-element simulations shows that they have a spatially nonuniform mass sensitivity or error due to mass positioning from the free end of the cantilever to the middle of the cantilever (top image), whereas NMR demonstrates spatial nonuniformity of mass sensitivity or error due to mass position to be less from the center to the edge of the platform (bottom image). (b) A schematic of the NMR sensor with piezoresistive sensing component, (c) Distribution of $\Delta R / R$ on the surface of NMR with a dimension of $100 \mu \mathrm{m} \times 5 \mu \mathrm{m}$ when a compressive force of $-1.0 \mathrm{nN}$ is applied uniformly, (d) Scanning electron micrograph (SEM) of NMR array chip with two different dimensions of $100 \mu \mathrm{m} \times 5 \mu \mathrm{m}$ and $50 \mu \mathrm{m} \times 5 \mu \mathrm{m}$ which are fabricated in the same array

where $R_{0}$ is the isotropic resistivity of the unstressed crystal, $\sigma_{i}$ is the stress component, and the term $\pi_{i j}$ is the component of the piezoresistance tensor. According to Eq. (1), for plain stress (i.e., $\sigma_{z}=0$ ), the relative resistance change can be described as follows:

$$
\frac{\Delta R}{R_{0}} \approx \frac{\pi_{44}}{2}\left(\sigma_{x}-\sigma_{y}\right)
$$

From Eq. (2), it is clear that $\Delta R / R_{0}$ is completely dependent on $\sigma_{x}$ and $\sigma_{y}$ values. In cantilever sensors, surface stress induces an isotropic stress, and the piezoresistive signal is nearly zero except at the clamped end where the isotropic symmetry is broken. Thus, the sensor sensitivity efficiently reduces in comparison with cantilevers when a point force is applied at the free end. According to this problem, the nanomechanical-membrane approach was presented by Yoshikawa et al. [18] for surface-stress detection. In the present work, the piezoresistive nanomechanical-membrane approach was developed for monitoring the resonant-frequency shift. A simple illustration of the 
Citation: Rahimpour A, Yazdian F, Jonoush ZA, Rajabibazl M, Omidi M (2016) Design and Manufacturing of Electromechanical Chip for Rapid Measurement of IgG1 Antibody in Cell-Culture Supernatant. Biol Med (Aligarh) 8: 340. doi:10.4172/0974-8369.1000340.

Page 3 of 5

NMR sensor with a piezoresistive sensing component can be observed in Figure 1b. Owing to Eq. (2), the isotropic surface stress leads to zero piezoresistive signal, but in the N-methylmorpholine (NMM) structure, the isotropic deformation effectively converts into a concentrated force at the connection between the membrane and the piezoresistive sensing component. Figure $1 \mathrm{c}$ shows the $\Delta R / R_{0}$ distribution for NMR with a dimension of $100 \times 5 \mu \mathrm{m}$, when a compressive force of $-1.0 \mathrm{~N}$ is applied uniformly on the NMR. The membrane-type geometry allows us to place a full Wheatstone bridge on the chip, and when all four resistors are practically equal, and the relative resistance changes are small, the total output signal $V_{\text {out }}$ can be approximated by:

$$
V_{\text {out }}=\frac{V_{\text {in }}}{4}\left(\frac{\Delta R_{1}}{R_{1}}-\frac{\Delta R_{2}}{R_{2}}+\frac{\Delta R_{3}}{R_{3}}-\frac{\Delta R_{4}}{R_{4}}\right)
$$

According to Eqs. (1)-(3), the average value of the relative resistance change in the NMR has a higher value in comparison with the standard cantilever (about 43 times). The intrinsic noise level for the modified piezoresistor can be estimated by Johnson (thermal) and Hooge (1/f) noise equations [24-26]. The total intrinsic noise for NMR is reported as $0.01-0.5 \mu \mathrm{V}[18,27]$, which is still lower than the experimental noises $(2.0 \sim 2.5 \mu \mathrm{V})$, mainly caused by the electrical circuit.

\section{Materials and Methods}

\section{Fabrication of NMR sensor}

We used Silicon on Insulator (SOI) wafers with a $2-\mu \mathrm{m}$ device layer and a $0.3-\mu \mathrm{m}$ buried oxide (BOX) layer as the substrate material. Then a $25-\mathrm{nm}$ silicon dioxide layer was grown by thermal oxidation to electrically insulate the device layer from the subsequent metal layers. The first lithographic process to define the first metal layer for the electrode and sensor platform for subsequent liftoff process has been accomplished. After patterning, the photoresist, chrome $(10 \mathrm{~nm})$, and gold $(50 \mathrm{~nm})$ layers were deposited by e-beam evaporator and patterned by a liftoff process with the previously patterned photoresist. The patterned metal layer from previous step and the patterned layer of photoresist from the second photolithographic process were used to define the areas to be etched to define the sensor structure. The exposed device layer was etched completely by reactive-ion etching (RIE) to define the sensor structure. Then, a third photolithographic step for the second liftoff process, followed by the deposition of a 30-nm chrome layer and a 150-nm gold layer for wire-bonding pads. After the liftoff, a release window was photolithographically defined by the fourth lithographic process, and the exposed BOX was etched by RIE, leaving the silicon substrate exposed.

Then the wafer was diced into individual chips. Through the release window, the exposed Si substrate was etched by vapor-phase etching using xenon difluoride $\left(\mathrm{XeF}_{2}\right)$ to release the sensor structure. After $\mathrm{XeF}_{2}$ etching, the photoresist and the BOX were removed by buffered hydrofluoric acid (BHF) etching and solvent cleaning. The die was cleaned with oxygen plasma, and then a 100-nm thick silicon dioxide layer was deposited with plasma enhanced chemical vapor deposition (PECVD) for insulation. The PECVD oxide on the bonding pads was selectively etched for wire bonding. Then each die was attached to a custom-made printed circuit board (PCB) and was wire bonded. Figure 1d presents the final picture of NMRs in different sizes fabricated in the same array using a scanning electron micrograph (SEM). NMRs with two different diameters of 50 and $25 \mu \mathrm{m}$ are fabricated in the same array. The sensor operates in a first-resonance mode, where the platform vibrates vertically at approximately $3.9 \mathrm{MHz}$ in air and approximately
1.4 MHz in liquid. Our sensor shows a mass sensitivity of $25 \mathrm{~Hz} / \mathrm{pg}$ in air and $1.8 \mathrm{~Hz} / \mathrm{pg}$ in liquid. The sensor was actuated by a Lorentz force generated by passing an actuation current through the sensor in a static magnetic field.

\section{Protein A immobilization process}

For cleaning the NMRs, the NMRs were settled for $30 \mathrm{~min}$ in a 2:1 piranha solution (a mixture of concentrated sulfuric acid and $30 \%$ hydrogen peroxide) and then for another $30 \mathrm{~min}$ in an oxygen plasma. A moisture-free environment was used to functionalize the devices with 3-aminopropyltriethoxysilane (APTES, Sigma, 99\%) overnight $(\sim 16 \mathrm{~h})$ using a $10 \%$ solution in dry toluene (Sigma, 99.8\%). Following silanization, the cantilevers were washed in a series of acetone, isopropanol, and water, and then in order to remove excess APTES, they were soaked in deionized (DI) water for $15 \mathrm{~min}$ on an orbital shaker. Moreover, for creating a covalent cross-linker molecule between the amine groups on the silanized surface and antibodies, chips were soaked in a $5 \%$ solution of gluteraldehyde (Sigma, 50\%) in borate buffer for $2 \mathrm{~h}$. Following this and all subsequent steps, device chips were washed twice, each washing step was for $2 \mathrm{~min}$, in purified DI water on an orbital shaker operating at $95 \mathrm{rpm}$. It should be mentioned that fresh water was used between washes. The reason for using water instead of buffer in the washing process was to prevent the abundant formation of buffer-salt crystals on the surface of devices, which make the sensors effectively useless.

Next, a 1-h incubation was used to immobilize the Protein A (Abcam, Inc), affinity-purified, with a concentration of $50 \mathrm{mg} / \mathrm{mL}$ on the surface. By immersing the NMR for $30 \mathrm{~min}$ in $50 \mathrm{mM}$ solution of glycine, unreacted gluteraldehyde was then quenched. In addition, bovine serum albumin (BSA, Sigma, St. Louis, MO, USA) dissolved in phosphate buffered saline (PBS) with $10 \mathrm{mg} / \mathrm{ml}$ concentration was used to prevent nonspecific binding. For this purpose, the NMRs were immersed in this solution for $1 \mathrm{~h}$ at room temperature. Then, they were rinsed with $\mathrm{PBS}$ ( $\mathrm{pH}$ 7.4) containing polyoxyethyethylenesorbitan monolaurate (Tween 20, St. Louis, MO, USA), and finally washing was performed by using only PBS solution.

\section{Electrical measurements}

For the electrical measurement of the sensor, internal DC-bias Wheatstone bridge was used. A bridge-supply voltage of $1.5 \mathrm{~V}$ was applied using a DC power supply (Agilent, E3631A), and the sensor's output voltage was measured by a multimeter (keithley, 2010 7-1/2). Moreover, a Faraday cage was adopted for noise reduction. The above components were used to measure the piezoresistive response of the NMR in a liquid environment.

\section{Test procedure}

The IgG1 antibody was purified with the HSA-PBS solution using D-Salt Excellulose Desalting Columns to remove the vendor-added solvent. The final solution was aliquoted and diluted to concentrations covering several orders of magnitude extending from 0.04 to 10,000 $\mathrm{ng} / \mathrm{ml}$. The control (such as BSA-PBS, HSA-PBS, HP-PBS) or analyte (IgG1 antibody) was then injected into the NMR-sensor arrays using a flexible polydimethylsiloxane (PDMS) polymer microfluidic channel sealed to the device chip. All the experiments were carried out at controlled temperature of $25.0 \pm 0.05^{\circ} \mathrm{C}$. The reaction happened in a static environment by molecular diffusion to the NMR surface and then by binding to the probes. The diffusion coefficient of the solute molecules is $d=k_{b} T / X$, where $k_{b}$ is the Boltzmann constant $\left(1.38 \times 10-{ }^{23} \mathrm{~J} / \mathrm{K}\right)$ 
Citation: Rahimpour A, Yazdian F, Jonoush ZA, Rajabibazl M, Omidi M (2016) Design and Manufacturing of Electromechanical Chip for Rapid Measurement of IgG1 Antibody in Cell-Culture Supernatant. Biol Med (Aligarh) 8: 340. doi:10.4172/0974-8369.1000340.

Page 4 of 5

$T$ is the absolute temperature, and $X$ is the frictional coefficient of the molecule given as $f=6 \pi \mu\left(3 V_{h} / 4 \pi\right)^{1 / 3}$. Here, $\mu$ is the viscosity of the solvent $\left(8.55 \times 10^{-4} \mathrm{Ns} / \mathrm{m}^{2}\right), V_{h}=M\left(V_{2}+\delta_{1} V_{1}\right) / N_{0}$ is the volume of the hydrated molecule, $M$ is the molecular weight of the solute molecule with the units $\mathrm{g} / \mathrm{mol}, N_{0}$ is Avogadro's number, $\delta_{1}$ is the hydration (grams of $\mathrm{H}_{2} \mathrm{O}$ bound per gram of solute, for protein, $\delta \approx 0.3$ ), $V_{1}$ is the partial specific volume of $\mathrm{H}_{2} \mathrm{O}\left(\approx 1 \mathrm{~cm}^{3} / \mathrm{g}\right)$, and $V_{2}$ is the partial specific volume of the solute (typical values for proteins: $0.69-0.75 \mathrm{~cm}^{3} / \mathrm{g}$ ). For the IgG1 antibody, $M=34,000 \mathrm{~g} / \mathrm{mol}, d=8.5 \times 10^{-7} \mathrm{~cm}^{2} / \mathrm{s}$.

\section{Results and Discussion}

In order to study the effect of nonspecific adsorption on the assay, we tested the detection of $5 \mathrm{ng} / \mathrm{ml}$ IgG1 antibody diluted in PBS and different concentrations of HSA serum (Figure 2). For both media, a control chip was used with no spiked IgG1 antibody present in order to determine the background effects of the buffer and serum. The primary issue that arises when working with serum rather than standard buffer solution is the nonspecific binding of other background proteins or biomolecules to the sensor surface. This is typically assumed to sterically block specific binding sites and reduce the amount of captured analyte. In addition, nonspecifically bound materials can alter the signals of sensors, potentially reducing or even exaggerating measured signals that should be associated with only specific interactions. As shown in Figure 2, according to the nonspecific adsorption a significant increase in background was observed for the chips exposed to HSA, but a large part of the nonspecifically bounding can be removed by using prolonged washing after serum injection.

Additional IgG1-antibody concentrations ranging from $100 \mathrm{pg} / \mathrm{ml}$ to $10 \mu \mathrm{g} / \mathrm{ml}$ in a HSA background were tested, and the frequency responses are shown in Figure 3a. Using a $100 \mu \mathrm{m} \times 5 \mu \mathrm{m}$ NMR, the lowest IgG1-antibody concentration that we could clearly detect above noise was $0.1 \mathrm{ng} / \mathrm{ml}$. Also shown in Figure $3 \mathrm{a}$ is the frequency response as a function of the IgG1-antibody concentration with $1 \mathrm{mg} / \mathrm{ml} \mathrm{HSA}$ as background. The experiment shows that the NMR sensor present required resolution for the IgG1-antibody detection.

With the use of the calibration curves for each NMR, IgG1antibody concentrations for the three unknown mixtures were determined. Figure $3 \mathrm{~b}$ displays the concentrations of the unknowns as determined using the NMR sensors. Each reported value is the

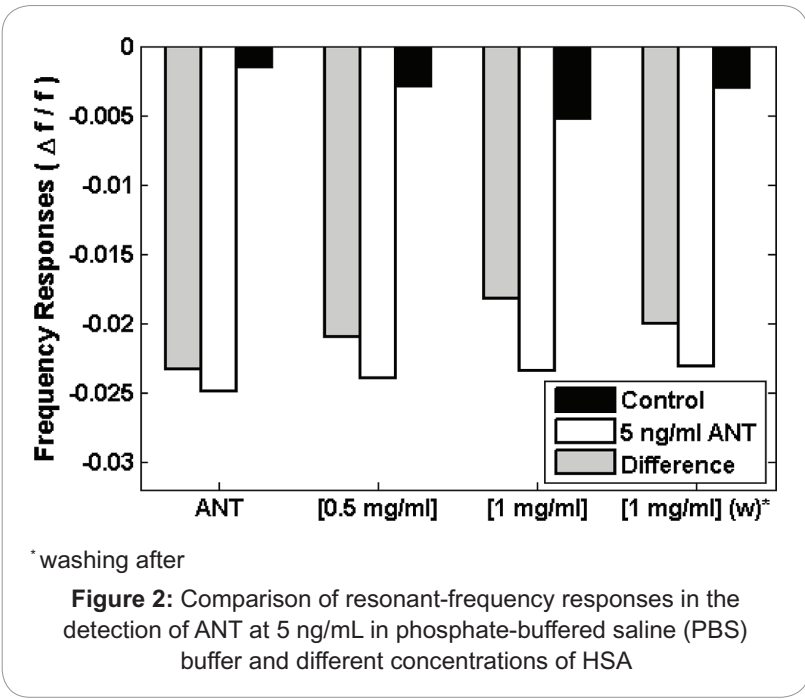

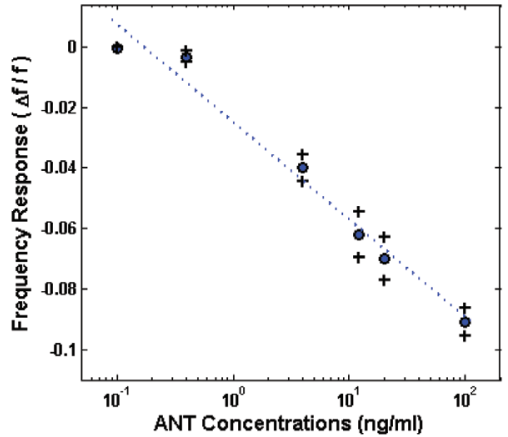

(a)
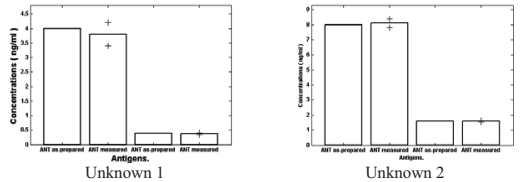

(b)

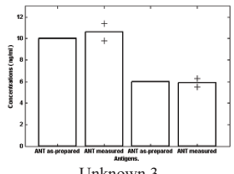

Unknown 3

Figure 3: (a) Frequency response of NMR sensors as a function of ANT concentrations. Every data point on this plot represents an average of output signals obtained in multiple experiments done with different NMR, whereas the range of output signals obtained from these experiments is shown as the error bar. (b) Evaluation of unknown protein cocktails 1, 2, and 3 comparing

the measured values with the actual, as-prepared values. Error bars represent the $95 \%$ confidence interval; $n=4$ trials

\begin{tabular}{|c|c|c|}
\hline Category & Detection conditions & LOD \\
\hline NMR & $0.1 \mathrm{mg} / \mathrm{ml} \mathrm{HSA}$ & $0.4 \mathrm{ng} / \mathrm{ml}$ \\
\hline $\begin{array}{c}\text { MCL [28] with reference cantilever, } \\
\text { piezoresistive detection }\end{array}$ & $0.1 \mathrm{mg} / \mathrm{ml} \mathrm{BSA}$ & $10 \mathrm{ng} / \mathrm{ml}$ \\
\hline $\begin{array}{c}\text { MCL [4] no reference cantilever, } \\
\text { optical detection }\end{array}$ & $1.0 \mathrm{mg} / \mathrm{ml} \mathrm{HSA}$ & $0.2 \mathrm{ng} / \mathrm{ml}$ \\
\hline SPR [30] Direct immunoassay & $0.3 \mathrm{mg} / \mathrm{ml} \mathrm{BSA}$ & $300 \mathrm{ng} / \mathrm{ml}$ \\
\hline $\begin{array}{c}\text { QCM [30] Direct assay based on } \\
\text { yeast cells strategy }\end{array}$ & Serum & $5 \mathrm{ng} / \mathrm{ml}$ \\
\hline $\begin{array}{c}\text { Electrochemical [31] Amperometric } \\
\text { Sandwich immunoassay }\end{array}$ & Phosphate buffer & $0.25 \mathrm{ng} / \mathrm{ml}$ \\
\hline
\end{tabular}

Table 1: The minimum limit of detection (LOD) of IgG1 antibody by different biosensors

average concentration as determined by four NMR sensors. With the comparison of these concentrations with the as-prepared values, it is apparent that all of the as-prepared concentrations fall within the error bounds of the measurement technique (95\% confidence interval, four independent measurements). The average measurement error was approximately $0.1 \mathrm{ng} / \mathrm{ml}$

In order to check the sensitivity of the present NMR-based biosensor, the results have been compared with other label-free biosensing technologies. In Table 1, the minimum IgG1-antibody detection limits (LODs) of NMRs have been compared with a standard cantilever (Microcantilever [MCL]) [4,14], surface-plasmon resonance (SPR) [29], quartz crystal monitor (QCM) [30], and electrochemical [31] sensors. In most cases, the NMR-based biosensor has the lowest LOD. Results indicate that NMR has comparable sensitivity with the optical readout methods; moreover its sensitivity is significantly higher than a standard piezoresistive cantilever. The large surface area of NMR is significantly greater than that of the standard cantilevers of 
Citation: Rahimpour A, Yazdian F, Jonoush ZA, Rajabibazl M, Omidi M (2016) Design and Manufacturing of Electromechanical Chip for Rapid Measurement of IgG1 Antibody in Cell-Culture Supernatant. Biol Med (Aligarh) 8: 340. doi:10.4172/0974-8369.1000340.

Page 5 of 5

the same length, thus increasing the probability of capturing analytes at extremely low concentrations, which may improve the sensitivity. In addition, if a cantilever with the same surface area as NMR were used, roughly the same amount of antigen would be bound to it, but the highly nonuniform frequency response of cantilevers along their length would likely increase the standard deviations for each concentration by a significant amount and also reduce the sensitivity. The assay by a standard cantilever could be improved when all frequency measurements are taken in vacuum, which presents some challenges to use in a clinical-laboratory setting. Experimental results show that the NMR sensors' devices could be used with high sensitivity in liquid in the pharmaceutical- and medical-diagnosis fields.

\section{Conclusion}

We used a unique array of piezoresistive nanomechanical-membrane resonators (NMR)-mass sensors for identifying the IgG1 antibody. Unlike the traditional cantilever geometry, NMR's frequency response is approximately independent of the location of the mass. Such a uniform response across much of the sensing area would be desirable for the detection of materials added in dilute concentrations, as it would reduce variations from device to device caused by randomly distributed binding events. On the other hand, the NMM-integrated piezoresistive readout sensors facilitate the detection of compact devices in even nontransparent environments. The unique NMR design significantly improves sensor sensitivity that allows us to detect IgG1-antibody concentrations as low as $400 \mathrm{pg} / \mathrm{ml}$. The high sensitivity of these robust NMR arrays, in addition to their small size and versatility, suggests that they will find use in many applications, including miniaturised sensors and multiplexed detection systems.

\section{References}

1. Alvarez M, Lechuga LM (2010) Microcantilever-based platforms as biosensing tools. Analyst 135(5): 827-836.

2. Omidi M, Alaie S, Rousta A (2012) Analysis of the vibrational behavior of the composite cylinders reinforced with non-uniform distributed carbon nanotubes using micro-mechanical approach. Meccanica 47(4): 817-833.

3. Baniasadi L, Omidi M, Amoabediny G, Yazdian F, Attar H, et al. (2014) An inhibitory enzyme electrode for hydrogen sulfide detection. Enzyme Microb Technol 63: 7-12.

4. Wu G, Datar RH, Hansen KM, Thundat T, Cote RJ, Majumdar A (2001) Bioassay of prostate-specific antigen (PSA) using microcantilevers. Nat Biotechnol 19(9): 856-860.

5. Omidi M, Malakoutian MA, Choolaei M, Oroojalian F, Haghiralsadat F, Yazdian F (2013) A Label-Free Detection of Biomolecules Using Micromechanical Biosensors. Chin Phys Lett 30(6): 068701.

6. Ghatkesar MK, Lang HP, Gerber C, Hegner M, Braun T (2008) Comprehensive characterization of molecular interactions based on nanomechanics. PLoS One 3(1): e3610.

7. Lang HP, Baller MK, Berger R, Gerber C, Gimzewski JK, et al. (1999) An artificial nose based on a micromechanical cantilever array. Anal Chim Acta 393(1): 59-65.

8. Thundat T, Warmack RJ, Chen GY, Allison DP (1994) Thermal and ambientinduced deflections of scanning force microscope cantilevers. Appl Phys Lett 64(21): 2894-2896.

9. Aeschimann L, Meister A, Akiyama T, Chui B W, Niedermann P, et al. (2006) Scanning probe arrays for life sciences and nanobiology applications. Microelectron Eng 83(4): 1698-1701.
10. Arlett JL, Maloney JR, Gudlewski B, Muluneh M, Roukes ML (2006) Selfsensing micro-and nanocantilevers with attonewton-scale force resolution. Nano Lett 6(5): 1000-1006.

11. Boisen A, Thundat T (2009) Design fabrication of cantilever array biosensors Mater Today 12(9): 32-38.

12. Mukhopadhyay R, Sumbayev VV, Lorentzen M, Kjems J, Andreasen PA Besenbacher $F$ (2005) Cantilever sensor for nanomechanical detection of specific protein conformations. Nano Lett 5(12): 2385-2388.

13. Rashidi A, Omidi M, Choolaei M, Nazarzadeh M, Yadegari A, et al. (2013) Electromechanical properties of vertically aligned carbon nanotube. In Advanced Materials Research (Vol. 705, pp. 332-336). Trans Tech Publications.

14. Privorotskaya NL, King WP (2009) The mechanics of polymer swelling on microcantilever sensors. Microsyst Technol 15(2): 333-340.

15. Omidi M, Choolaei M, Asjodi F, Haghiralsadat F, Yazdian F (2014) Measuremen of Prostate Specific Antigen Using Self-sensing Nanomechanical Membrane. Procedia Eng 87: 660-663.

16. Loui A, Goericke FT, Ratto TV, Lee J, Hart BR, King WP (2008) The effect of piezoresistive microcantilever geometry on cantilever sensitivity during surface stress chemical sensing. Sens Actuators A 147(2): 516-521.

17. Yang SM, Yin TI, Chang C (2007) Development of a double-microcantilever for surface stress measurement in microsensors. Sens Actuators B 121(2): 545-551.

18. Yoshikawa G, Akiyama T, Gautsch S, Vettiger P, Rohrer H(2011) Nanomechanica membrane-type surface stress sensor. Nano Lett 11(3): 1044-1048.

19. Waggoner PS, Varshney M, Craighead HG (2009) Detection of prostate specific antigen with nanomechanical resonators. Lab Chip 9(21): 3095-3099.

20. de Caprona DC, Fritzsch B, Camens AB, Phillips MJ, Bennett TH, et al. (2010) Dog genome evolution: A strategy to segregate biogeographic effects from human selection. PNAS 107(4): 1255-1256.

21. Dohn S, Sandberg R, Svendsen W, Boisen A (2005) Enhanced functionality of cantilever based mass sensors using higher modes. Appl Phys Lett 86(23): 233501.

22. Berger R, Delamarche E, Lang HP, Gerber C, Gimzewski JK, et al. (1997) Surface stress in the self-assembly of alkanethiols on gold. Science 276(5321): 2021-2024.

23. Tufte ON, Stelzer EL (1963) Piezoresistive properties of silicon diffused layers J Appl Phys 34(2): 313-318.

24. Hooge FN (1969) $1 / f$ noise is no surface effect. Phys Lett $A$ 29(3): 139-140.

25. Harley JA, Kenny TW (1999) High-sensitivity piezoresistive cantilevers under 1000 Å thick. Appl Phys Lett 75(2): 289-291.

26. Yu X, Thaysen J, Hansen O, Boisen A (2002) Optimization of sensitivity and noise in piezoresistive cantilevers. J Appl Phys 92(10): 6296-6301.

27. Yoshikawa G, Akiyama T, Loizeau F, Shiba K, Gautsch S, et al. (2012) Two dimensional array of piezoresistive nanomechanical membrane-type surface stress sensor (MSS) with improved sensitivity. Sensors 12(11): 15873-15887.

28. Wee KW, Kang GY, Park J, Kang JY, Yoon DS, et al. (2005) Novel electrical detection of label-free disease marker proteins using piezoresistive selfsensing micro-cantilevers. Biosens Bioelec 20(10): 1932-1938.

29. Besselink GA, Kooyman RP, van Os PJ, Engbers GH, Schasfoort RB (2004) Signal amplification on planar and gel-type sensor surfaces in surface plasmon resonance-based detection of prostate-specific antigen. Anal Biochem 333(1) 165-173.

30. Ding Y, Lu H, Shi G, Liu J, Shen G, Yu R (2008) Cell-based immobilization strategy for sensitive piezoelectric immunoassay of total prostate specific antigen. Biosens Bioelec 24(2): 228-232.

31. Sarkar P, Pal PS, Ghosh D, Setford SJ, Tothill IE (2002) Amperometric biosensors for detection of the prostate cancer marker (PSA). Int J Pharm 238(1): 1-9. 